\title{
Good News from Africa
}

\author{
Soziale Arbeit in Afrikas Region der Großen Seen
}

Soziale Arbeit in Afrikas Region der Großen Seen hat mit großen sozialen Problemen zu kämpfen und ist auch als Profession mit beträchtlichen strukturellen Herausforderungen konfrontiert. Während in der Ausbildung importierte Theorien und Konzepte aus Europa und Nordamerika dominieren, haben in der Praxis zumeist internationale Fördergeber das Sagen. Ein internationales Projekt hatte die Professionalisierung Sozialer Arbeit in einigen dieser Länder zum Ziel.

I $\mathrm{n}$ den westlichen Medien und europäischen Köpfen wird Afrika zumeist einseitig als „K-Kontinent“ dargestellt und wahrgenommen: Kontinent der Kriege, Katastrophen, Krisen, Krankheiten und Korruption. Tatsächlich handelt es sich um einen bunten Kontinent mit 54 Staaten und einer beträchtlichen geographischen, klimatischen, kulturellen, sozialen, politischen und ökonomischen Vielfalt. Die Lebenswelten der Menschen sehen entsprechend sehr unterschiedlich aus. Während der Großteil der Bevölkerung nach wie vor auf dem Land lebt, verzeichnen einige afrikanische Staaten gleichzeitig die höchsten Urbanisierungsraten der Welt (Hove et al. 2013). In den Großstädten gibt es immer mehr Slums, die von Armut und Gewalt geprägt sind. Unterdessen kristallisiert sich, bedingt durch ein steigendes Wirtschaftswachstum, langsam eine schmale Mittelschicht mit einem gehobeneren Lebensstandard heraus. Der Alltag der Bevölkerung wird sukzessive von Modernisierungserscheinungen durchdrungen. Vor allem das Mobiltelefon und das Internet haben die Lebensgewohnheiten der Menschen maßgeblich verändert.

\begin{tabular}{l|l|} 
Helmut Spitzer \\
Feldkirchen, Österreich \\
*1966; FH-Prof. Mag. Dr., Professor für Soziale Arbeit an der \\
Fachhochschule Kärnten. Langjährige Forschungserfahrung in \\
Ostafrika. \\
h.spitzer@fh-kaernten.at
\end{tabular}

Zusammenfassung In der afrikanischen Region der Großen Seen kämpft Soziale Arbeit in Abgrenzung zu importierten Theorien und Konzepten um ein eigenständiges, indigenes Profil. Dazu lief über mehrere Jahre ein Projekt zur Erforschung und Stärkung der Profession. Zentrale Komponenten dieses Projekts waren Forschung, Lehrplanentwicklung, Berufspolitik und Lobbyarbeit.

Schlüsselwörter Afrika, Indigenisierung, Internationale Soziale Arbeit, Professionalisierung, Soziale Entwicklung
Die großen sozialen Probleme in Subsahara-Afrika sind - bei aller Vorsicht, was Verallgemeinerungen betrifft absolute Armut, soziale Ungleichheit, hohe Arbeitslosigkeit (vor allem bei der jungen Generation), fehlende soziale Sicherheit, Fluchtbewegungen, Menschenrechtsverletzungen sowie politische Gewalt, die sich in einigen Ländern in Form bewaffneter Konflikte manifestiert. Hinzu kommen gravierende Auswirkungen des Klimawandels, beispielsweise durch anhaltende Dürreperioden, die besonders in Ostafrika zu Nahrungsmittelkrisen geführt haben (Society for International Development 2016, S. 21).

Soziale Arbeit stellt sich als heterogenes und komplexes Feld dar, mit länderspezifischen Unterschieden, aber auch einigen Gemeinsamkeiten (Spitzer 2018): In den meisten Ländern ist Soziale Arbeit im Zuge der kolonialen Sozialplanung und in weiterer Folge nach der Unabhängigkeitsbewegung in den 1960er-Jahren durch die sogenannte Entwicklungshilfe als fremdbestimmte Profession entstanden (Midgley 1981). Diese von James Midgley (ebd.) als „professioneller Imperialismus“ titulierte Überformung mit westlich orientierten Theorien, Konzepten und Methoden in Ausbildung und Praxis dauert tendenziell bis heute an.

\section{Große Seen, große Herausforderungen}

Folgende Länder werden zur Region der Großen Seen (dazu gehören vor allem der Viktoriasee und der Tanganjikasee) gezählt: Burundi, die Demokratische Republik Kongo, Kenia, Ruanda, Tansania und Uganda. Mit Ausnahme von Tansania haben diese Länder eine gewaltvolle Geschichte hinter sich, die von ethnischen Konflikten, Bürgerkrieg und Völkermord geprägt ist. Besonders der Genozid in Ruanda, bei dem 1994 bis zu einer Million Menschen ermordet wurden, hatte weitreichende Folgen und führte zur Destabilisierung der gesamten Region und zum Zerfall des vormaligen Staates Zaire, der nun Demokratische Republik Kongo heißt (vgl. Spitzer und Twikirize 2014). Ruanda ist weltweit 
das einzige Land, in dem professionelle Soziale Arbeit als Reaktion auf einen Völkermord und die völlige Zerstörung eines Landes ins Leben gerufen worden ist.

Angesichts dieser Problemlagen hat Soziale Arbeit enorme Herausforderungen zu meistern und als Profession selbst mit einer Reihe von Strukturproblemen zu kämpfen. Zunächst gibt es viel zu wenig Ausbildungsstätten: In den Ländern der Ostafrikanischen Gemeinschaft (Burundi, Kenia, Ruanda, Tansania und Uganda) mit einer Bevölkerung von 170 Mio. gibt es insgesamt nur 44 tertiäre Bildungseinrichtungen für Soziale Arbeit (Spitzer 2019, S. 571). Zum Vergleich: Allein in Deutschland gibt es mehr als 150 facheinschlägige Studiengänge. Die meisten Curricula an den afrikanischen Hochschulen sind stark westlich orientiert, zudem gibt es einen großen Mangel an fachlich qualifiziertem Lehrpersonal. Forschung zu Sozialer Arbeit war bis vor wenigen Jahren so gut wie inexistent. Und für die Praxis kann man die Arbeitsrealität wohl in einem Satz zusammenfassen: Zu wenige Sozialarbeiter_innen müssen sich um zu viele Probleme und Klient_innen kümmern, bekommen obendrein zu wenig bezahlt und erhalten kaum gesellschaftliche Anerkennung. Bei einer in vier ostafrikanischen Ländern durchgeführten Studie kam heraus, dass mehr als die Hälfte der befragten Sozialarbeiter_innen $(51 \%)$ im Non-Profit-Sektor arbeitet (Twikirize et al. 2014), und dieser wird fast ausschließlich von internationalen Fördergebern und Spendengeldern finanziert. Mit anderen Worten: Die Praxis der Sozialen Arbeit ist von großer ausländischer Abhängigkeit geprägt. Wenn kein Geld mehr fließt, kommen auch die Projektaktivitäten zum Stillstand.

\section{Trotz allem gute Neuigkeiten}

Was also sind die im Titel dieses Beitrags angekündigten „Good News“? Ich beziehe mich dabei auf ein Projekt internationaler Sozialer Arbeit, das als Antwort auf die skizzierten Strukturprobleme in diesen Ländern von 2010 bis 2019 in mehreren Phasen durchgeführt wurde. Es handelt sich dabei um eine Kooperation zwischen mehreren ostafrikanischen Hochschulen und der Fachhochschule Kärnten in Österreich, das die Erforschung, Stärkung und Professionalisierung Sozialer Arbeit zum Ziel hatte (Spitzer 2019). Die zentralen Komponenten des Projekts PROSOWO (Promotion of Professional Social Work) waren Forschung, Lehrplanentwicklung, Berufspolitik (Stärkung der Berufsverbände für Soziale Arbeit) und Lobbyarbeit (sozialpolitische Einflussnahme und Menschenrechtsarbeit).

In Phase I (2010) wurden in einem partizipativen Prozess die Ziele, Aktivitäten und Meilensteine des Projekts auf Basis der von den afrikanischen Kolleg_innen artikulierten Bedarfslagen konzipiert. Dazu trafen sich die Projektpartner_innen zu einer einwöchigen Klausur in Nairobi, Kenia. In Phase II (2011 bis 2014) konzentrierte sich das Projekt auf Grundlagenforschung, die Überarbeitung der Curricula und berufspolitische Aspekte. Die Partnerorganisationen waren die University of Nairobi in Kenia, die University of Rwanda, das Institute of Social Work in Tansania und die Makerere University in Uganda. In Phase III (2016 bis 2019) kamen die Hope Africa University in Burundi sowie der neu gegründete Berufsverband für Soziale Arbeit in der Demokratischen Republik Kongo an Bord. Dabei lag der Schwerpunkt auf der Erforschung indigener und innovativer Praxisansätze sowie auf dem sozialpolitischen und menschenrechtlichen Engagement Sozialer Arbeit. Das Projekt wurde durch das Programm APPEAR (Austrian Partnership Programme in Higher Education and Research for Development) der Österreichischen Entwicklungszusammenarbeit finanziert. Durch eine Reihe von Publikationen, neue Curricula, mehrere Fachkonferenzen sowie einen intensiven Dialog mit politischen Entscheidungsträgern konnten einige nachhaltige Akzente zur Stärkung Sozialer Arbeit in den betroffenen Ländern gesetzt werden.

\section{Von Grundlagenforschung zu angewandter Praxisforschung}

In Phase II wurde eine umfassende Studie zum Status quo Sozialer Arbeit in Ausbildung, Praxis und Berufspolitik sowie zum Beitrag der Profession zur Armutsbekämpfung und sozialen Entwicklung durchgeführt. Auf Basis einer aus quantitativen und qualitativen Forschungsmethoden zusammengesetzten Befragung von 2000 Personen (Sozialarbeiter_innen, Lehrende, Studierende, Arbeitgeber, politisch Verantwortliche und Klient_innen) liegen nun erstmals empirische Daten zu Sozialer Arbeit in diesen Ländern vor (Spitzer et al. 2014). In Phase III wurden im Zuge angewandter Praxisforschung indigene, d. h. kulturspezifisch relevante und innovative Ansätze der Lebensbewältigung und Problemlösung beforscht. Die Ergebnisse aus 155 qualitativen Interviews und 55 Fokusgruppendiskussionen zeigen vor allem eines deutlich: Die indigene Praxis der Sozialen Arbeit ist stark kollektiv ausgerichtet und fokussiert ihre Interventionen in erster Linie auf die Community und weniger auf das Individuum (Twikirize und Spitzer 2019).

Dazu zwei Beispiele: In der Region Kilimanjaro in Tansania arbeiten Sozialarbeiter_innen in einigen Communities mit dem sogenannten Msaragambo-System. Dieser Begriff aus der Volksgruppe der Chagga bezieht sich auf lokal organisierte, von Dorfältesten geführte Selbst- 


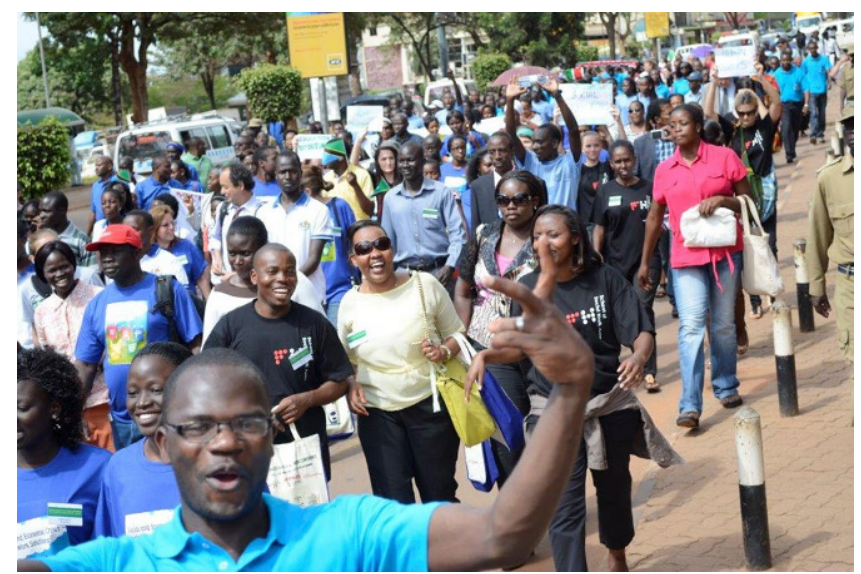

Abb. 1 "Sozialarbeiter_innen marschieren für soziale Gerechtigkeit" (C) Decent Africa

hilfegruppen, die bei der Feldarbeit, bei finanziellen Engpässen und der Bearbeitung von sozialen Konflikten Unterstützung bieten. Obwohl sich die Praxis durch Land-Stadt-Migration und moderne Einflüsse stark gewandelt hat, kommt sie nach wie vor zum Einsatz, beispielsweise bei der Unterstützung von Witwen, Waisenkindern und alten Menschen (Mabeyo et al. 2019).

Ein weiteres Beispiel kommt aus Uganda: Dort wurde im Westen des Landes eine Organisationsform namens Bataka identifiziert, die als Hilfsmaßnahme bei Beerdigungen zum Tragen kommt. Durch die hohen Infektionsraten mit AIDS sind Familien und Gemeinschaften mit häufigen Todesfällen konfrontiert. Diese bringen neben der emotionalen Belastung auch ökonomische Schwierigkeiten mit sich. Die Bataka-Gruppen unterstützen Hinterbliebene im Trauerprozess, aber auch durch Geldleistungen, da Beerdigungen oft mit hohen Kosten verbunden sind. Sie sind somit wichtige Resilienzfaktoren im Gemeinwesen (Twesigye et al. 2019).

Solche kulturspezifischen Bewältigungsformen stellen für die Praxis der Sozialen Arbeit wertvolle Anknüpfungspunkte dar, besonders vor dem Hintergrund einer fast völligen Abwesenheit staatlicher Sozialleistungen. Auf die Bedeutung indigenen Wissens wird auch in der internationalen Definition Sozialer Arbeit hingewiesen (International Federation of Social Workers 2015, S. 19). Doch die Berufung auf kulturelle Werte und traditionelle Praktiken sollte nicht unkritisch erfolgen. Auch die Aufrechterhaltung patriarchaler Gesellschafts- und Familienstrukturen und die damit einhergehende Unterdrückung und Benachteiligung von Mädchen und Frauen wird kulturell legitimiert. Ein drastisches Beispiel dafür ist die weibliche Genitalverstümmelung, die bei einigen ethnischen Gruppen in Ostafrika als sozial sanktionierter Initiationsritus im Übergang vom Kind zur erwachsenen Frau praktiziert wird (vgl. Spitzer 2006, S. 80 ff.). Soziale Arbeit muss sich hier fachlich und ethisch positionieren, aber gleichzeitig muss sie sich mit kulturspezifischen Normen und Traditionen in kritisch-reflexiver Weise auseinandersetzen und sich auf einen dialogischen Prozess einlassen, der mühsam und manchmal zermürbend ist.

\section{Afrikanische Curricula für afrikanische Lebenswelten}

Die empirischen Erkenntnisse aus beiden Forschungsphasen wurden in einer Reihe von Fachbüchern publiziert und durch ein strategisches Disseminationsverfahren allen Ausbildungsstätten für Soziale Arbeit in den teilnehmenden Ländern zugänglich gemacht. Das war ein bedeutsamer Schritt, zumal in den ostafrikanischen Hochschulbibliotheken überwiegend Fachliteratur aus dem außerafrikanischen Ausland vorherrscht (vgl. Twikirize et al. 2014, S. 208). Somit steht Lehrenden und Studierenden ein authentisches Lern- und Diskussionsmaterial zur Verfügung.

Bei den beteiligten Hochschulen wurden auf Basis der Forschungsergebnisse die bestehenden Curricula für Bachelor-Studiengänge überarbeitet. Diese im Fachdiskurs als Indigenisierung oder auch Dekolonialisierung bezeichnete Entrümpelung und Neugestaltung der Lehrpläne ist ein entscheidender Schritt in der sozialberuflichen Qualifizierung: Die Studierenden müssen bereits auf der Ausbildungsebene mit kultur- und kontextspezifischen Wissensinhalten in Berührung kommen, um auf die jeweiligen Realitäten ihrer späteren Berufspraxis adäquat vorbereitet zu sein (Mwansa 2010). Die Partnerhochschulen in Tansania und Uganda entwickelten darüber hinaus neue Lehrpläne für ein Master-Studium in Sozialer Arbeit, wobei ebenfalls empirisch basierte, indigene Wissenselemente eingeflossen sind.

\section{Social Development als transformative Soziale Arbeit}

Die theoretische Fundierung für viele Methoden und Konzepte, die für afrikanische Kontexte geeignet sind, findet sich im sogenannten Social Development-Ansatz Sozialer Arbeit (Chitereka 2009). Dieser spielte auch bei dem Projekt PROSOWO eine zentrale Rolle. Dabei geht es im Kern um eine Akzentuierung Sozialer Arbeit, die in ihrem Interventionsspektrum vorwiegend auf mesound makrostrukturelle Ebenen abzielt, um dadurch Armut und soziale Benachteiligung zu bekämpfen und den Lebensstandard der Menschen anzuheben. Basierend auf Prinzipien sozialer Gerechtigkeit, der Menschenrechte, Inklusion, Partizipation und Freiheit soll durch einen initiierten sozialen Wandel das Wohlergehen der Gesamtbevölkerung in Verbindung mit einem dynami- 
schen Entwicklungsprozess gefördert werden (Midgley 2010). Dadurch bekommt Soziale Arbeit einen deutlich politischen und transformativen Charakter. In Kombination mit genuin afrikanischen Wissenssystemen wird im Social Development-Ansatz die Möglichkeit eines Paradigmenwechsels gesehen (Chitereka 2009), bei dem die Hypotheken von Kolonialismus und Entwicklungshilfe endlich abgestreift werden können.

\section{Politisches Lobbying und sozialer Aktivismus}

Eine wesentliche Komponente des Projekts PROSOWO war die Einflussnahme auf politische Entscheidungsinstanzen, allen voran die zuständigen Ministerien. $\mathrm{Zu}$ diesem Zweck wurden mehrere länderübergreifende Vernetzungstreffen sowie zahlreiche nationale Meetings und Workshops durchgeführt. In Kenia, Tansania und Uganda konnten durch diese Aktivitäten und in Kooperation mit dem jeweiligen nationalen Berufsverband erste Schritte in Richtung eines Gesetzesentwurfs zur Regulierung und Lizenzierung Sozialer Arbeit erreicht werden.

Auch Öffentlichkeitsarbeit und Bewusstseinsbildung für die Belange Sozialer Arbeit und ihrer Zielgruppen standen auf der Agenda des Projekts. In diesem Zusammenhang wurde im Zuge mehrerer internationaler Konferenzen, die im Zeitraum von 2014 bis 2018 allesamt am World Social Work Day (dritter Dienstag im März) stattfanden, auf sozialen Aktivismus gesetzt. Höhepunkt dieser Events, die in Kampala (Uganda), Bujumbura (Burundi), Arusha (Tansania) und Kigali (Ruanda) stattfanden, war jeweils ein öffentlichkeitswirksam inszenierter Marsch für Soziale Arbeit, soziale Gerechtigkeit und den Schutz der Menschenrechte (s. Abb. 1 und 2).

Das kann in einem afrikanischen Kontext durchaus politische Brisanz haben bzw. sogar gefährlich sein. Im März 2014 marschierten im Rahmen einer internationalen Fachkonferenz an die 500 Sozialarbeiter_innen auf den Straßen der ugandischen Hauptstadt Kampala und demonstrierten dabei u. a. für die Rechte sexueller Minderheiten. Das war zu einem Zeitpunkt, als in diesem Land gerade ein Gesetzesentwurf zur Einführung der Todesstrafe für homosexuelle Menschen verhandelt wurde. Nach der Konferenz wurde der ugandische Berufsverband monatelang von Regierungsvertretern schikaniert und bekam ein vorübergehendes Betätigungsverbot ausgesprochen.

\section{Das Prinzip Hoffnung am Beispiel von Ost-Kongo}

Der Osten der Demokratischen Republik Kongo ist seit Jahrzehnten Schauplatz kriegerischer Auseinandersetzungen und grausamer Menschenrechtsverletzungen.

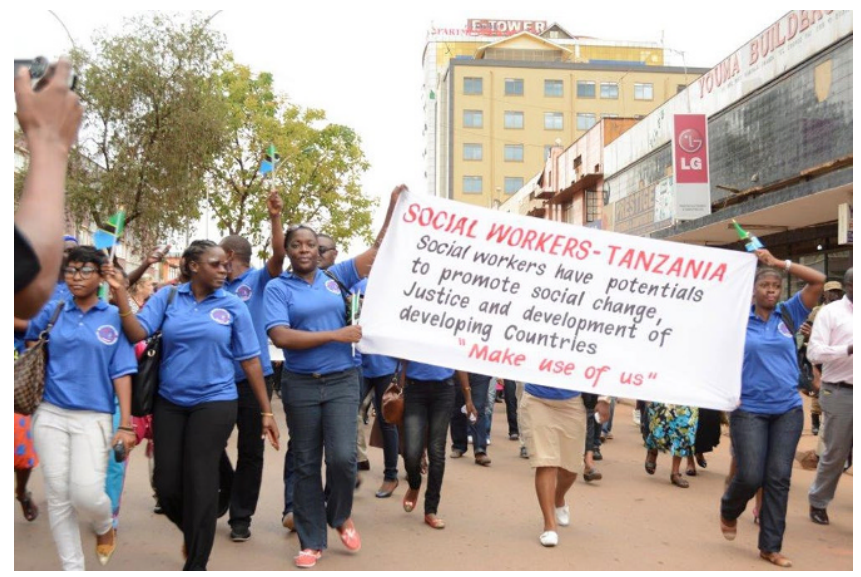

Abb. 2 "Tansanische Sozialarbeiter_innen machen auf sich aufmerksam" () Decent Africa

Der Alltag der Menschen ist von einer omnipräsenten Kultur der Gewalt geprägt. Ein kongolesischer Sozialarbeiter hat dies bei einer Konferenz im Zuge einer Diskussion über Menschenrechte und Ethik einmal so auf den Punkt gebracht: „Bei uns gibt es keine Ethik.“ Mit dieser Aussage verdichtete er das moralische Vakuum in einem Kontext, in dem beinahe täglich Menschen getötet und Frauen vergewaltigt werden. Diese andauernde Kultur der Gewalt ist tief mit der traumatischen Kolonialgeschichte des Landes verstrickt (vgl. Van Reybrouck 2013).

Angesichts chronischer Armut ist ein Großteil der Bevölkerung einem ständigen Überlebenskampf ausgesetzt. Dabei gilt der Ost-Kongo als eine der ressourcenreichsten Regionen der Welt mit großen Vorräten an Bodenschätzen wie Öl, Gold, Diamanten und Coltan. Letzteres ist ein Erz, das für die Herstellung von Handys benötigt wird und entsprechende Begehrlichkeiten bei internationalen Konzernen hervorruft, die die Region in großem Stil ausbeuten.

Professionelle Soziale Arbeit steckt im Ost-Kongo erst in den Kinderschuhen. Die meisten Sozialarbeiter_innen haben ihre Qualifikation in einem der Nachbarländer erworben. Seit 2013 gibt es an der Université Evangélique en Afrique in der Stadt Bukavu erstmals einen Studiengang für Soziale Arbeit. 2018 gelang es einer Gruppe von hoch motivierten Sozialarbeiter_innen, trotz immenser bürokratischer, logistischer und finanzieller Hürden, einen Berufsverband für Soziale Arbeit zu gründen. Dieser dient als Plattform für Öffentlichkeitsund Lobbyarbeit, für die schwierige und manchmal auch gefährliche Kommunikation mit Politik, Exekutive und Militär, sowie für vereinzelte Projektaktivitäten, die besonders vulnerablen Bevölkerungsgruppen zugutekommen sollen. Dazu zählt z. B. Gefängnissozialarbeit. Die Gefängnisse sind völlig überfüllt, die Insassen leiden 
vielfach an Krankheiten und Unterernährung, und immer wieder kommt es zu Gewaltausbrüchen und sexuellen Übergriffen. Bei regelmäßigen Besuchen wird den eingesperrten Menschen psychosoziale Beratung angeboten, und wenn genügend Geld vorhanden ist, versucht das Sozialarbeitsteam, Nahrungsmittel, Medikamente und Hygieneartikel zur Verfügung zu stellen.

Der Einsatz der Sozialarbeiter_innen in der Krisenregion des Ost-Kongo ist nur ein Beispiel dafür, dass es möglich ist, trotz extrem schwieriger Rahmenbedingungen konkrete Verbesserungen in der Lebenssituation der Menschen herbeizuführen. Ich selbst hatte in den letzten 20 Jahren viele Gelegenheiten, mit Kolleg_innen in einigen afrikanischen Ländern zusammenzuarbeiten, und ich bin immer wieder beeindruckt von ihrem Engagement, Optimismus und Humor. Während in Europa tendenziell auf hohem Niveau gejammert wird, dominiert auf unserem südlichen Nachbarkontinent die Hoffnung auf eine bessere Zukunft.

Funding. Open access funding provided by Carinthia University of Applied Sciences (CUAS).

Open Access. Dieser Artikel wird unter der Creative Commons Namensnennung 4.0 International Lizenz veröffentlicht, welche die Nutzung, Vervielfältigung, Bearbeitung, Verbreitung und Wiedergabe in jeglichem Medium und Format erlaubt, sofern Sie den/die ursprünglichen Autor(en) und die Quelle ordnungsgemäß nennen, einen Link zur Creative Commons Lizenz beifügen und angeben, ob Änderungen vorgenommen wurden.

Die in diesem Artikel enthaltenen Bilder und sonstiges Drittmaterial unterliegen ebenfalls der genannten Creative Commons Lizenz, sofern sich aus der Abbildungslegende nichts anderes ergibt. Sofern das betreffende Material nicht unter der genannten Creative Commons Lizenz steht und die betreffende Handlung nicht nach gesetzlichen Vorschriften erlaubt ist, ist für die oben aufgeführten Weiterverwendungen des Materials die Einwilligung des jeweiligen Rechteinhabers einzuholen.

Weitere Details zur Lizenz entnehmen Sie bitte der Lizenzinformation auf http://creativecommons.org/licenses/by/4.0/deed.de.

\section{Literatur}

Chitereka, C. (2009). Social work practice in a developing context: The case of Africa. Advances in Social Work, 10(2), 144-156.

Hove, M., Ngwerume, E. T., \& Muchemwa, C. (2013). The urban crisis in sub-Saharan Africa: A threat to human security and sustainable development. Stability, 2(1), 1-14.

International Federation of Social Workers (IFSW) (2015). IFSW's policies 2015. Bern: IFSW.

Mabeyo, Z. M., Mvungi, A., \& Manyama, W. (2019). Community organising in Tanzania: Learning from the Msaragambo model in Kilimanjaro region. In J. M. Twikirize \& H. Spitzer (Hrsg.), Social work practice in Africa. Indigenous and innovative approaches (S. 111-124). Kampala: Fountain.

Midgley, J. (1981). Professional imperialism: Social work in the Third World. London: Heinemann.
Midgley, J. (2010). The theory and practice of developmental social work. In J. Midgley \& A. Conley (Hrsg.), Social work and social development. Theories and skills for developmental social work (S. 3-28). Oxford: Oxford University Press.

Mwansa, L.-K. J. (2010). Challenges facing social work education in Africa. International Social Work, 53(1), 129-136.

Society for International Development (2016). State of East Africa report. Consolidating misery? The political economy of inequalities in East Africa. Nairobi: SID.

Spitzer, H. (2006). Kinder der Straße. Kindheit, Kinderrechte und Kinderarbeit in Tansania. Frankfurt a.M.: Brandes \& Apsel.

Spitzer, H. (2019). Social work in East Africa. A Mzungu perspective. International Social Work, 62(2), 567-580.

Spitzer, H., \& Twikirize, J. M. (2014). Armed conflict and political violence in Africa's Great Lakes Region. Challenges for social work education and practice. In H. Spitzer, J. M. Twikirize \& G. G. Wairire (Hrsg.), Professional social work in East Africa. Towards social development, poverty reduction and gender equality (S. 351-369). Kampala: Fountain.

Spitzer, H. (2018). Soziale Arbeit und soziale Entwicklung in Afrika. In H.-U. Otto, et al. (Hrsg.), Handbuch Soziale Arbeit. Grundlagen der Sozialarbeit und Sozialpädagogik (S. 1448-1457). München: Reinhardt.

Spitzer, H., Twikirize, J. M., \& Wairire, G. G. (Hrsg.). (2014). Professional social work in East Africa. Towards social development, poverty reduction and gender equality. Kampala: Fountain.

Twesigye, J., Twikirize, J. M., Luwangula, R., \& Kitimbo, S. (2019). Building resilience through indigenous mechanisms: The case of Bataka groups in Western Uganda. In J. M. Twikirize \& H. Spitzer (Hrsg.), Social work practice in Africa. Indigenous and innovative approaches (S. 145-160). Kampala: Fountain.

Twikirize, J. M., Spitzer, H., Wairire, G. G., Mabeyo, Z. M., \& Rutikanga, C. (2014). Professional social work in East Africa: Empirical evidence. In H. Spitzer, J. M. Twikirize \& G. G. Wairire (Hrsg.), Professional social work in East Africa. Towards social development, poverty reduction and gender equality (S. 189-216). Kampala: Fountain.

Twikirize, J. M., \& Spitzer, H. (Hrsg.) (2019). Social work practice in Africa. Indigenous and innovative approaches. Kampala: Fountain.

Van Reybrouck, D. (2013). Kongo. Eine Geschichte. Bonn: Bundeszentrale für politische Bildung. 
Hier steht eine Anzeige.

\section{Springer}

